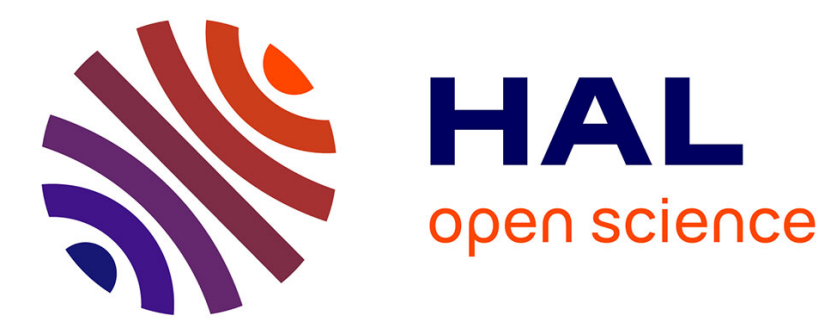

\title{
A comparison between liquid drops and solid particles in partial wetting
}

\author{
Antonio Stocco, Maurizio Nobili
}

\section{To cite this version:}

Antonio Stocco, Maurizio Nobili. A comparison between liquid drops and solid particles in partial wetting. Advances in Colloid and Interface Science, 2017, 247, pp.223-233. 10.1016/j.cis.2017.06.014 . hal-01627865

\section{HAL Id: hal-01627865 https://hal.science/hal-01627865}

Submitted on 11 Mar 2019

HAL is a multi-disciplinary open access archive for the deposit and dissemination of scientific research documents, whether they are published or not. The documents may come from teaching and research institutions in France or abroad, or from public or private research centers.
L'archive ouverte pluridisciplinaire HAL, est destinée au dépôt et à la diffusion de documents scientifiques de niveau recherche, publiés ou non, émanant des établissements d'enseignement et de recherche français ou étrangers, des laboratoires publics ou privés. 


\title{
A comparison between liquid drops and solid particles in partial wetting
}

\author{
Antonio Stocco* and Maurizio Nobili \\ Laboratoire Charles Coulomb (L2C), UMR 5221 CNRS-Univ. Montpellier, Montpellier F-34095, France.
}

\begin{abstract}
In this critical review we compare two geometries in partial wetting: a liquid drop on a planar substrate and a spherical particle at a planar liquid interface. We show that this comparison is far from being trivial even if the same physical interactions are at play in both geometries. Similarities and differences in terms of free energies and frictions will be discussed. Contact angle hysteresis, the impact of surface roughness and line pinning on wetting will be described and compared to selected experimental findings.
\end{abstract}

\section{Introduction}

Wetting properties of surfaces are fundamental in many research and industrial fields such as coating, microfluidics, thin films, microelectronics, and in membrane and cell biology [1],[2],[3],[4]. Affinity and interactions between a liquid and a surface can be quantified by a spreading coefficient or an equilibrium contact angle, which defines whether a liquid completely wets a surface or not [5]. Equilibrium contact angle $\alpha_{e q}$ provides already a clear insight into the wetting properties of a surface with a given liquid, but $\alpha_{e q}$ alone describes only a small part of the wetting properties of a surface. Surface structure and interactions at different length scales dramatically impact static and dynamic wetting properties, which may lead to stable, metastable and unstable contact angle regimes [5,6]. It is important to note that in some cases, a thermodynamic equilibrium contact angle could be never observed due to kinetic effects. Viscous, molecular frictions, and contact line pinning result from the dissipations occurring during the relative motion of the liquid and the solid [7]. Thus, the slow motion of the contact line due to these large dissipations may also lead to observable contact angles, which are far from the thermodynamic equilibrium. These frictions lead to intriguing wetting scenarios, which deserve dedicated investigations in order to achieve clear insights into the wetting properties of a surface. Now, it is important to underline that wetting is not restricted to planar surfaces and solid-liquid-gas systems. In this article we consider also a spherical solid surface geometry like the one of small particles. Solid spherical-like particles like grains or nanoparticles are also strongly impacted by wetting in many fields. In flotation, solid spherical particles can be separated by adsorption onto gas bubbles due to their wettability [8]. Because of partial wetting, particles are also used to obtain very stabilized foams and emulsions, called Pickering emulsions [9],[10]. In phase transfer, like in liquid-liquid extraction, particles are required to adsorb onto an interface and after undergoing a complete wetting-dewetting dynamics are able to transfer into a target phase [11]. To address all these phenomena, many experimental and theoretical investigations on colloids and nanoparticles at the liquid-gas or liquid-liquid interfaces have been carried out for many years [12-20]. Some unexpected experimental results have been also found, including: (i) discrepancies between the contact angle attained by a particle at a planar liquid interface and the contact angle of a liquid drop on a substrate made of the same particle material [21],[22] ; (ii) unexpected slow lateral diffusion coefficients 
of solid particles straddling liquid interfaces [23],[24],[17]. It is worth noting that when dealing with small particles at liquid interfaces, partial wetting is often reduced to the simple concept of a particle equilibrium contact angle $\alpha_{e q}$. Rarely, contact angle hysteresis, contact line dynamics and roughness effects are investigated and discussed. This critical review aims at bridging the wetting knowledge obtained for liquid drops at planar solid surface and the wetting of spherical particles at the liquid interface.

Here we would like also to highlight how rich the wetting of micro- and nano- particles is, and the potentials of these particles as probes in partial wetting. In fact, different length scales and interactions can be investigated and explored by tuning the size and the surface properties of particles.

\subsection{Free energy, force and equilibrium contact angle for planar and spherical surfaces}

We start this review by a description in an ideal world where the surfaces are perfectly smooth and defect free, see Figure 1. We focus on drop or particle sizes smaller than the capillary length $l_{c}=\sqrt{\gamma / \rho g} \approx 2 \mathrm{~mm}$ (where $\gamma$ is the surface tension of the liquid $\rho$ is the liquid density and $g$ is the gravitational acceleration) but larger than a characteristic length scale of long range surface forces $l_{s}=\alpha_{e q}^{-2} \sqrt{H / 6 \pi \gamma} \approx 1 \ldots 10 \mathrm{~nm}$ (where $\alpha_{e q}$ is the equilibrium contact angle, $H$ is the Hamaker constant) [25]. We also assume that the liquid and the solid are not volatile. Throughout this article we will compare the behaviour of spherical solid particle of radius $R$ and a liquid drop having the same radius, initially, when it touches the solid substrate, $R_{0}\left(\alpha=180^{\circ}\right)=R$. Hence, the volume of the drop is kept constant for any contact angles, see Fig. 1. Moreover, here we neglect deformations of the planar solid and liquid interface. Under these assumptions, a spherical liquid drop touching an infinite solid substrate will search for an equilibrium contact angle, keeping the same volume but changing its shape and area. In the size range considered here, the liquid drop adopts a spherical cap profile according to the Laplace equation. A solid particle touching an infinite liquid interface will also search an equilibrium contact angle but it will keep both its volume and area constant, see Figure 1.

\section{Figure 1}

Equilibrium position of the liquid drop can be found calculating the free energy of the system as a function of the contact angle $\alpha$. At the point of contact the drop radius is $R_{0}$ and the free energy is $E\left(\alpha=180^{\circ}\right)=\sigma_{S G} A+\gamma 4 \pi R_{0}^{2}$, where $A$ is the planar surface area and $\sigma_{S G}$ is the solid-gas interfacial tension (Figure 1). Note that $\sigma_{\mathrm{SG}}$ and $\gamma$ can be defined as excess interfacial free energies per unit area [26]. For liquid and solid interfaces, the liquid-gas surface tension $\gamma$ and the solid-fluid interfacial tension $\sigma_{\mathrm{i}}$ are the derivatives of the free energy with respect to the area (at constant temperature $T$, total volume $V$ and number of molecules $n$ ), $\gamma$ or $\sigma_{i}=\left(\frac{d E}{d A_{i}}\right)_{T, V, n}$ [27]. At an arbitrary contact angle $\alpha$, the free energy of the system is: 
$E(\alpha)=\sigma_{S G} A-\sigma_{S G} A_{0}+\sigma_{S L} A_{0}+\gamma A_{L G}$

Where $\sigma_{S L}$ is the solid-liquid interfacial tension. $A_{0}$ is the area covered by the drop, $A_{0}=\pi R_{0}(\alpha)^{2} \sin ^{2} \alpha$. $A_{L G}$ is the liquid-gas interfacial area $A_{L G}=2 \pi R_{0}(\alpha)^{2}(1-\cos \alpha)$ and $R_{0}(\alpha)=V^{\frac{1}{3}}\left[\frac{\pi}{3}(2-3 \cos \alpha+\right.$ $\left.\left.\cos ^{3} \alpha\right)\right]^{-\frac{1}{3}}$.The drop base radius is $R_{d}=R_{0}(\alpha) \sin \alpha$, see Figure 1a.

The thermodynamic equilibrium contact angle can be defined at the position where $E(\alpha)$ is minimum, see Fig. 2. This minimum is described by the Young-Laplace equation:

$\cos \alpha_{e q}=\left(\sigma_{S G}-\sigma_{S L}\right) / \gamma$

Note that in the limit of zero contact angle, the free energy tends to infinity. In this limit, a finite value of the energy will be set by the long-range surface force at play. The liquid may adopt the form of a film (molecular or nanometric) or liquid pancake [28].

For a spherical particle at a fluid interface the same approach can be used, see Figure 1b. The calculation starts considering a colloid completely dry, which touches a free planar liquid interface. The free energy of the system (colloid plus free interface) is simply given by the product of the areas and the corresponding interfacial tensions: $E\left(\alpha=180^{\circ}\right)=\gamma A+\sigma_{S G} 4 \pi R^{2}$, where $R$ is the radius of the spherical particle and $A$ is the area of the free interface. When the solid particle crosses the interface, a solid-liquid interface is created, the energy of the solid-gas interface is reducing and the liquid-gas interface is missing an area $A_{0}$ due to the particle adsorption. The free energy of the system as a function of the particle position expressed as $\cos \alpha=-z / R$ is ( $z$ is the vertical distance between the center of the particle and the interface: $z>0$ in gas) [29]:

$E(\alpha)=\gamma A+2 \pi R^{2}\left[\sigma_{S G}(1-\cos \alpha)+\sigma_{S L}(1+\cos \alpha)-\frac{1}{2} \gamma \sin ^{2} \alpha\right]$

Note that besides wetting no additional interactions are accounted in equation Eq. 3.

The thermodynamic equilibrium contact angle can be defined at the position where the $E(\alpha)$ is minimum, see Figure 2:

$\left(\sigma_{S G}-\sigma_{S L}\right) / \gamma=-z_{0} / R=\cos \alpha_{e q}$

Equation 4 is equivalent to Equation 2. Equilibrium of forces acting on the contact line of a partially wetted colloid at a fluid interface or a liquid drop partially wetting a solid substrate leads always to: $\sigma_{S G}=$ $\sigma_{S L}+\gamma \cos \alpha_{e q}$

Figure 2 
If the drop or the particle is at a position $\alpha$ different from $\alpha_{e q}$, the system is out of equilibrium. The imbalance due to $\alpha \neq \alpha_{e q}$ produces a force $F_{l}=-\frac{d E}{d s}$, where $s$ represents the displacement of the contact line on the solid, acting on the triple line which is proportional to the contact line length.

For a liquid drop, the displacement of the contact line is radial and parallel to the solid plane (see inset in Figure 2B). Using the convention $F_{l}>0$ for $\alpha>\alpha_{\text {eq }}$, the force acting on the line $F_{l}$ is:

$$
F_{l}(\alpha)=2 \pi R_{d} \gamma\left(\cos \alpha_{e q}-\cos \alpha\right)=2 \pi R_{0}(\alpha) \sin \alpha \gamma\left(\cos \alpha_{e q}-\cos \alpha\right)
$$

For a spherical particle, the displacement of the contact line is the longitudinal coordinate $s=R \theta$ ( $\theta$ is the polar angle in spherical coordinates (see inset in Figure 2B). Using the convention $F_{l}>0$ for $\alpha>\alpha_{\mathrm{eq}}$, the force $F_{l}$ acting tangential to the solid surface is:

$F_{l}(\alpha)=2 \pi R \sin \alpha \gamma\left(\cos \alpha_{e q}-\cos \alpha\right)$.

Given that $R_{0}$ is a function of $\alpha$, for liquid drops the force $F_{l}$ (equation 5 ) tends to diverge for very low contact angles, see Figure 2. Instead for spherical particles, the force must be zero when the length of the contact line is null: $\alpha=0$ and $180^{\circ}$ (equation 6), see Figure 2. If we consider a drop and a process leading to the equilibrium from a very low contact angle, the force will be high at low angles $(\alpha \rightarrow 0)$ and smoothly decreases when approaching $\alpha_{e q}$. Whereas for a particle, the force acting during a path from low $\alpha$ to $\alpha=$ $\alpha_{e q}$ shows a non-monotonic behavior, which is qualitatively the same behavior experienced during the path from $\alpha=180^{\circ}$ to $\alpha=\alpha_{e q}$ for both drop and particle. Hence, it becomes clear that wetting and dewetting dynamics may be very different for drops and particles. The two dynamics may be similar for the path from $\alpha=180^{\circ}$ to $\alpha=\alpha_{e q}$, which is encountered in a typical advancing contact angle experiment. The force $F_{l}$ reflects the changes of the wetted perimeter and the imbalance of the liquid-gas interfacial tension. $F_{l}$ shows a maximum corresponding to the large increase of the wetted perimeter at high angles, which is then counterbalanced by the decreasing liquid-gas interfacial tension component. Finally, one could calculate the vertical force acting on a spherical particle as $F_{p}=-F_{l}(\alpha) \sin \alpha$, which shows the same qualitative behaviour as the one discussed previously (equation 6).

\subsection{Line tension and length scale dependent contact angle}

In Figure 3, we show a local contact angle profile for a drop on a solid accounting for attractive Van der Waals interactions of Hamaker constant $H$. Note that at the nanometric scale $l_{s}=\alpha_{e q}^{-2} \sqrt{H / 6 \pi \gamma} \approx 1 \ldots 10 \mathrm{~nm}$ the drop profile deviates from the spherical cap profile calculated accounting only for constant surface tension.

Even if the exact profile of the contact angle at length scale $l<l_{s}$ is not known, in both drop and particle geometries, a thermodynamic equilibrium contact angle $\alpha_{e q}$ can be always defined [5]. Assuming that the local contact angle profile simply translates in the nanoscale when the drop is shifted by a distance $\mathrm{d} x$, see Figure 3, the work associated to an incremental area change $\mathrm{d} x \mathrm{~d} L$ ( $L$ is the length of the line considered) on the solid reads [5]:

$\sigma_{S G} \mathrm{~d} x \mathrm{~d} L-\sigma_{S L} \mathrm{~d} x \mathrm{~d} L-\gamma \mathrm{d} x \mathrm{~d} L \cos \alpha_{e q}=0$, 
which leads to the Young-Laplace equation.

Figure 3

If instead of assuming that the local contact angle profile simply translates when the drop is shifted by a distance $\mathrm{d} x$, one may introduce a line energy contribution $k \mathrm{~d} L$, where $k$ is the line tension. $k$ is defined as the energy per unit contact line length and represents the leading term of the total energy of the system after subtracting volume and interface contributions [31]. Hence, the line tension is the derivative of the free energy with respect to the contact line $L$ (at constant temperature $T$, total volume $V$, area, and number of molecules $n), k=\left(\frac{d E}{d L}\right)_{T, V, A, n}$ [32]. The addition of a line tension term in Equation 7 [33] leads to:

$\sigma_{S G} L \mathrm{~d} x-\sigma_{S L} L \mathrm{~d} x-\gamma L \mathrm{~d} x \cos \mathcal{A}_{e q}-k \mathrm{~d} L=0$

From Eq. (8) one finds $\sigma_{S G}=\sigma_{S L}+\gamma \cos \mathcal{A}_{e q}+k \mathrm{~d} L /(L \mathrm{~d} x)$, where $\mathcal{A}_{e q}$ is the equilibrium contact angle accounting for the line tension $k$.

$\cos \mathcal{A}_{e q}=\cos \alpha_{e q}-\frac{k}{\gamma} \frac{d L}{L d x}$

Note that in the partial wetting of a sessile drop, $\mathrm{d} L=2 \pi\left(R_{d}+\mathrm{d} x\right)-2 \pi R_{d}=2 \pi \mathrm{d} x$ (where $R_{d}$ is the droplet base radius $\left.R_{d}=R_{0}(\alpha) \sin \mathcal{A}_{e q}\right)$ and $\mathrm{d} L /(L \mathrm{~d} x)=1 / R_{d}$ is the curvature of the contact line:

$\cos \mathcal{A}_{e q}=\cos \alpha_{e q}-k /\left(\gamma R_{d}\right)$

$\cos \mathcal{A}_{e q}=\cos \alpha_{e q}-\frac{k}{\gamma R_{0}(\alpha)} \frac{1}{\sin \mathcal{A}_{e q}}$

The previous equation can be also obtained by the derivation of the free energy of the system (equation 11) as we have shown in the previous section:

$E(\alpha)=\sigma_{S G} A-\sigma_{S G} A_{0}+\sigma_{S L} A_{0}+\gamma A_{L G}+k 2 \pi R_{0}(\alpha) \sin \alpha$.

For solid spherical particles, when the line tension $k$ is accounted, the free energy becomes [34]:

$E(\alpha)=\gamma A+2 \pi R^{2}\left[\sigma_{S G}(1-\cos \alpha)+\sigma_{S L}(1+\cos \alpha)-\frac{1}{2} \gamma \sin ^{2} \alpha+\frac{k}{R} \sin \alpha\right]$.

For both drops and particles, positive (negative) $k$ means that an increase of the length of the contact line represents an energy cost (gain). For negative values of $k$ the minimum $E(\alpha)$ becomes deeper, whereas for positive values of $k$ the energy minimum could even disappear, see Figure 4. By deriving Equation 12 along the longitudinal coordinate, one calculates the force acting on the contact line:

$F_{l}(\alpha)=2 \pi R \gamma\left[\left(\cos \alpha_{e q}-\cos \alpha\right) \sin \alpha+\frac{k}{\gamma R} \cos \alpha\right]$. 
For $F_{l}=0$, the equilibrium angle can be found:

$\cos \mathcal{A}_{e q}=\cos \alpha_{e q}+\frac{k}{\gamma R} \frac{\cos \mathcal{A}_{e q}}{\sin \mathcal{A}_{e q}}$

It is important to remark the difference between Equation 10 and 14. For the droplet geometry and positive line tension, the contact angle will always increase in order to reduce the length of the contact line and the energy cost associated (keeping the volume of the droplet constant). For a spherical particle the decrease of the length of the contact line will depend on the immersion of the particle. A particle that is mostly located in the gas phase, in order to reduce the length of the contact line, will increase its contact angle; whilst a particle that is mostly immersed in the liquid will decrease its contact angle.

In Figure 4 we plot the free energies for drop and particle geometries for different values of line tension with $\alpha_{e q}=65^{\circ}$ as in Figure $2 \mathrm{a}$. In both geometries, negative line tensions lead to a deeper energy minimum. If the line tension value increases, one observes opposite shifts of the equilibrium contact angle $\mathcal{A}_{\text {eq }}$. Since positive line tensions represent an energy cost, energy minima could vanish and an energy maximum could appear. For the particle geometry, stable, metastable and unstable regions as a function of the strength of $k /(\gamma R)$ were described by Aveyard and Clint [34] and corrected by Drelich [18]. Note that by scrutinising equation 11, one would find stable, metastable and unstable regimes for droplets showing positive line tension as well. The existence of an energetic barrier against partial wetting may have serious consequences. Drop or particle with relative high line tensions may remain in a complete wetting or dry states simply due to the high energetic cost associated to the creation of the contact line.

\section{Figure 4}

Berg et al.[35] and Checco et al.[36,37] investigated the line energy effects for droplets of different dimensions down to the nanometer scale. They found negative values of $k \approx-\left(10^{-11} \ldots 10^{-10} \mathrm{~N}\right)$ and show the significance of the line energy contribution for droplet heights smaller than $20 \mathrm{~nm}$. Checco et al. noted that a constant line tension (i.e. length scale independent) is not able to described the obtained results and consider also the effect of line pinning [36,37]. Note that, the line tension, as it was introduced here, is not a source of contact angle hysteresis (see section 4). Here we keep the line tension as a purely thermodynamic quantity. In analogy with the interfacial tension for low energy system, which is of the order of magnitude of $k_{b} T / l_{m}{ }^{2} \approx 40 \mathrm{mN} / \mathrm{m}\left(l_{m}=0.3 \mathrm{~nm}\right.$ is a molecular length) [27], the order of magnitude of line tension can be estimated as $k_{b} T / l_{m} \approx 10^{-11} \mathrm{~N}$. It is worth noting that line tension $k \approx 10^{-11} \mathrm{~N} \ldots 10^{-12}$ have been usually measured in biphasic lipid monolayers [38,39]. For drops or particles, the line tension results from two main contributions. Short and long range forces such as Van der Waals and electrostatic forces represent the first contribution and they are usually included into the joining/disjoing pressure, which competes with the capillary pressure at the nanoscale [40]. The second contribution is represented by the increase (for repulsive interactions) or decrease (for attractive interactions) of the liquid-gas interfacial area with respect to the spherical cap profile (see Figure 3) [41][42]. It is important to recall that line tension could be also tuned by molecular or polymeric species present in the contact line region, which act as line active species $[43,44]$. 
It is quite clear that the magnitude of $k /(\gamma R)$ deserves a further discussion in order to illustrate the impact of positive line tensions. Only for very small particle of $10 \mathrm{~nm}$ radius (and for typical values of contact angles and interfacial tensions), a line energy $k \geq 10^{-10} \mathrm{~N}$ leads to the vanishing of the energy minimum across the interface [45]. Latter values of the line tension can be regarded as the upper bound limit value of physically meaningful line tension $[46,47]$. Hence, the line tension effect is expected to play a dramatic role only for spherical particle radii smaller than $10 \mathrm{~nm}$ or for particles with high aspect ratios [45]. This result may be of great importance for the mass transfer of nanoparticles across a fluid interface in liquidliquid extraction. As for droplets or bubbles in the submicron scale [48], also for particles some experimental results show indeed that the equilibrium contact angle changes with the size. Experiments on silica particles of radii between 70 and $500 \mathrm{~nm}$ showed systematic change of the contact angle at the liquid polystyrene-air interface [49]. Line tension $k \approx 10^{-9} \mathrm{~N}$ has been reported, which is somewhat higher than the theoretical expectation. High line tensions are usually suspected to account for dissipations rather than for energetic contributions. Note also that if line tension is introduced as a thermodynamic quantity, it does not imply any distribution of stable contact angles, i.e. $\mathcal{A}_{e q}$ is a single value. Isa et al. have measured the contact angle of different nanoparticles of radii between $10 \mathrm{~nm}$ and $1.4 \mu \mathrm{m}$ at the oil-water interface. They show a distribution of stable particle contact angles for each particle size. The width of the contact angle distribution becomes large if the particle size decreases. These important experimental findings have been discussed accounting for line tension $\left(k \approx 10^{-10} \ldots 10^{-9} \mathrm{~N}\right)$ and Brownian motion. One might wonder if the distribution of $\mathcal{A}_{e q}$ could result from stable and metastable contact angles; but in this case the contact angle distribution would be bimodal or ill-defined and rather high (see Figure 4B). Brownian motion could explain a distribution of stable contact angle for small nanoparticles but not for large particles. Hence, observation of particle contact angle distributions may deserve further analysis and discussion in terms of contact line pinning (see section 4) [50]. Finally, line tension can be seen as an excess quantity, which is the first order correction to the surface energy [35]. As shown in figure 3 for a liquid drop, the local profile in the vicinity of the contact line deviates from the spherical cap shape (described by the Young-Laplace equation) due to long-range surface forces [51],[30]. Two distinct profiles can be described. For $\alpha_{e q}<90^{\circ}$, attractive long range forces lead to local contact angles that increase to $90^{\circ}$ when approaching the contact line, see Figure 3. Repulsive long range forces lead to local contact angles which tend to zero at the nanoscale.[41] In the latter case, drops or particles are in equilibrium with a molecular film liquid adsorbed on the solid surface [25],[52,53]. For large drops or particles $R, R_{0}>0.1 \mu \mathrm{m}$, line tension effects are in general negligible and the equilibrium contact angle remains the same if the size of the system is increased. For small drops or particles $R, R_{0}<0.1 \mu \mathrm{m}$, equilibrium contact angle may become length scale dependent [30] and stable, metastable and unstable states could manifest. Note that reference [30] contains a mistake: attractive forces and repulsive forces should be interchanged [54].

\section{Roughness and inhomogeneity effects on Partial Wetting}

In order to describe partial wetting systems closer to the ones encountered in experiments, we should start describing the effects of surface defects. We start by considering the case of an homogeneous solid with roughness.

2.1 Roughness effects on Partial Wetting. Here, we assume that roughness is due to random topographical defects and could be modelled by geometrical functions. The main assumption here is that the liquid wets the solid such that no air trapping occurs, see Figure 5. Moreover the roughness is 
randomly distributed on the solid surface in a length scale much smaller than the system size. This approach is well-known in partial wetting of planar surface and named after "Wenzel" [55].

\section{Figure 5}

For a liquid drop, in presence of surface roughness, the surface $A_{s}$ of the solid-liquid and solid-gas interfaces becomes larger than the flat area $A_{0}, A_{s}=r A_{0}$, where $r$ is the ratio between the real area and the projected flat area. Thus the free energy becomes:

$E(\alpha)=\sigma_{S G} A-\sigma_{S G} A_{s}+\sigma_{S L} A_{s}+\gamma A_{L G} \quad$.

For a solid particle at a liquid-gas interface, decorated by defects as described before, the free energy reads:

$E(\alpha)=\gamma \mathrm{A}+2 \pi R^{2}\left[r \gamma_{S L}(1+\cos \alpha)+r \gamma_{S G}(1-\cos \alpha)-\frac{1}{2} \gamma \sin ^{2} \alpha\right]$

In order to illustrate the effect of roughness on wetting, we model the roughness as composed by cylindrical defects of radius $R_{s}$ and height $h_{s}$, which are homogenously present on the substrate with a surface fraction $\phi_{s}$.

$A_{s}=A_{0}\left(1+2 \phi_{s} \frac{h_{s}}{R_{s}}\right)$,

Where $\phi_{s}=n_{s} \pi R_{s}^{2}$ /(area of the solid surface) is the defect concentration ( $n_{s}$ is the number of defects) and the roughness is simply $r=\left(1+2 \phi_{s} \frac{h_{s}}{R_{s}}\right)$. In Figure 6 we plot the free energies for drop and particle geometries for different defect concentrations. Both for drop and particle geometries the energy minimum defines an equilibrium contact angle that follows the Wenzel equation [55]:

$\cos \alpha_{W}=r \cos \alpha$, where $r=A_{s} / A_{0}$.

\section{Figure 6}

Plotting the energy profiles for different defect concentrations at $h_{s} / R_{s}=1$, we note that for the drop geometry, an increase of $r$ leads to a deeper energetic minimum. On the contrary, for the solid particle the energy minimum becomes shallower and could even vanish if $r$ increases as for a particle decorated with surface defects covering $40 \%$ of the whole surface in Figure 6 [56]. This result is rarely accounted in the literature and has a clear impact on the location [57] and irreversibility of particle adsorption onto the surface. It is important to recall that the Wenzel equation is not valid at all length scales. For solid surface showing large structures $\left(l>l_{\mathrm{s}}\right)$, the liquid drop may "feel" an effective flat solid interface composed by the solid and the infiltrated liquid. The latter case corresponds to a Cassie-Baxter state. For larger surface 
structures and equilibrium contact angle $\alpha_{e q}>\alpha_{c}=\operatorname{arcos} \frac{1-\phi_{s}}{r-\phi_{s}}$, the Wenzel equation is valid. For $\alpha_{e q}<\alpha_{c}$ the Cassie-Baxter model introduced in the next section should be used [27].

\subsection{Inhomogeneity effects on Partial Wetting}

Beside roughness, a second scenario accounting for flat but heterogeneous solid surface can be discussed. If the solid surface is composed by two materials showing different surface energies randomly distributed on the whole surface, one refers to the "Cassie-Baxter" wetting state [27]. For planar solids with area fractions $\phi$ of materials 1 and $2, \phi_{1}+\phi_{2}=1$ :

$E(\alpha)=\phi_{1}\left(\sigma_{S I G} A-\sigma_{S I G} A_{0}+\sigma_{S I L} A_{0}\right)+\phi_{2}\left(\sigma_{S 2 G} A-\sigma_{S_{2} G} A_{0}+\sigma_{S 2 G} A_{0}\right)+\gamma A_{L G} \quad$.

At the energy minimum, the "Cassie-Baxter" contact angle is defined as [27]:

$\cos \alpha_{C B}=\phi_{1} \cos \alpha_{e q, 1}+\phi_{2} \cos \alpha_{e q, 2}$

Note that the previous equations are equivalent to the equations for homogenous solid surfaces if written in terms of effective surface energies, $\sigma_{S M G}=\sigma_{S 1 G} \phi_{1}+\sigma_{S 2 G} \phi_{2}$ and $\sigma_{S M L}=\sigma_{S I L} \phi_{1}+\sigma_{S 2 L} \phi_{2}$ :

$E(\alpha)=\sigma_{S M G} A-\sigma_{S M G} A_{0}+\sigma_{S M L} A_{0}+\gamma A_{L G}$

The same analysis applies to the spherical particle geometry. Two remarks can be now made. First, assuming that inhomogeneity is randomly distributed in a small scale compared to the system size we are always assuming that on average the contact line touches the two regions 1 and 2 with the same fraction as the area fraction. Note that if inhomogeneity is either on a scale comparable to the system size or structured in defined patterns, equations 19 and 20 do not hold anymore [58,59]. An exemplar case is represented by a drop sitting on a substrate with a single circular domain made of a different material [60]. If the drop sits inside the domain the equilibrium contact angle is the one of the material's domain. If the drop is larger than the domain and the domain is included in the drop, the equilibrium contact angle is the one of the substrate even if there is a large area fraction of the domain. For these particular cases where the heterogeneity length scale is large, it becomes clear that the relevant fractions $\phi$ in Equation 20 are not the area but the line fractions [60]. A long debate exists in the literature about this topic and the validity of Wenzel and Cassie-Baxter models [60],[61], [34-37],[66].

To further discuss the wetting of heterogeneous surfaces, and illustrate whether line or area fractions should be used in equation 20, we consider the cases of the partial wetting of Janus and patchy particles. A very rich behavior can be discussed when considering a Janus particle composed by two hemispherical faces with different contact angles at a liquid-gas interface [67,68]. If one Janus face possesses an equilibrium contact angle larger than $90^{\circ}$ and the other face lower than $90^{\circ}$, the free energy shows a minimum and a singular point at $90^{\circ}$, corresponding to a four-phase contact line where the two solid faces, the liquid and the gas meet [68]. If both faces show equilibrium contact angles lower (or higher) than $90^{\circ}$, stable, unstable and metastable wetting states can be described depending on the orientation of the particle, see Figure 7. When the Janus boundary is not crossing the liquid-gas, the contact line touches only one face of the Janus particle. When the contact line touches the face with the highest contact angle $\alpha_{e q, 1}$, the Janus particle finds a stable contact angle at $\alpha_{e q, 1}$ [69]. If, instead, the contact line touches the face with the lowest contact angle $\alpha_{e q, 2}$, the Janus particle may remain at metastable contact angle equal to 
$\alpha_{e q, 2}$ [56]. Note that in this geometry the heterogeneity has the same size of the particle and Cassie-Baxter model does not apply. If the Janus particle is oriented with the Janus boundary crossing the liquid-gas interface, no equilibrium can be found if the liquid-gas interface keeps flat [56],[70]. A different scenario can be discussed for patchy particles. If a patchy particle possesses randomly distributed regions of two materials of sizes much smaller than the particle size, the contact line will intimately touch both regions. In this case a Cassie-Baxter regime can be expected and the stable particle contact angle will be defined by Equation 20.

\section{Figure 7}

\section{Energy of planar solids and liquid interfaces covered by particles}

In this section we consider the surface energies of composite interfaces. We start with some simple considerations gained from the previous sections. We begin by considering the planar surface geometry. The total energy of a dry homogenous and flat solid surface is simply $E=\sigma_{S G} A$, see Figure 8a [5]. If the solid is rough, $E=\sigma_{S G} r A$ (see equation 15 and Figure $8 \mathrm{~b}$ ). If instead it is composed by two materials (see equation 20):

$E=\sigma_{S^{\prime} G} \phi_{1} A+\sigma_{S^{2} G} \phi_{2} A$.

Antonow's rule describes the energy of a system composed by a macroscopic liquid film on a solid surface in complete wetting [5,72]:

$E=\sigma_{S L} A+\gamma A$.

If the liquid film is not macroscopic, interaction between liquid-gas and solid-liquid interface should be accounted and the energy of the system is [27]:

$E=\sigma_{S L} A+\gamma A+P(h) A$

The Derjaguin joining/disjoining pressure $\Pi$ is related to $P(h)$ by $\Pi=-\mathrm{d} P / \mathrm{dh}$. Note that if $h$ is large, $P(h)$ $=0$ and we recover Equation 23, whereas if $h=0, P(h)=\sigma_{S G}-\sigma_{S L}-\gamma$ [27].

Now we turn our attention on the free energy of a liquid interface (Fig. 8d) in presence of solid spherical particles, Figure 8e. The total energy of the system (bulk plus interface) can be calculated as:

$E_{T O T}(\alpha)=\gamma A+N_{S} 2 \pi R^{2}\left[\sigma_{S G}(1-\cos \alpha)+\sigma_{S L}(1+\cos \alpha)-\frac{1}{2} \gamma \sin ^{2} \alpha\right]+N_{B} 4 \pi R^{2} \sigma_{S L}$

Where $N_{S}$ is the number of particles at the interface and $N_{B}$ is the number of particles in the volume: $N_{S}+$ $N_{B}=$ constant. Now we may ask if the number of particles in the bulk affects the free energy of a liquidgas interface covered by particles and the effective interfacial tension of the liquid-gas interface. The answer is clearly no because in a purely wetting perspective, particles are not surfactant molecules. For surfactants equilibrium between bulk and surface holds and the interfacial tension depends on the bulk concentration via the Gibbs equation. In a purely wetting perspective, particles in the bulk are not in 
equilibrium with the ones at the surface as the adsorption is irreversible and particles in the volume eventually sediment or float. Moreover, experiments pointed out that the interfacial tension measured for particle dispersions remains constant in absence of particle adsorption or in diluted interfacial regimes [9],[73,74]. The change of the effective interfacial tension measured in experiments seems to depend only on the lateral interactions between particles [75],[76]. Then, the interfacial energy of a liquid surface covered by particles is:

$$
\begin{aligned}
& E_{I}(\alpha)=\gamma A+N_{S} 2 \pi R^{2}\left[\sigma_{S G}(1-\cos \alpha)+\sigma_{S L}(1+\cos \alpha)-\frac{1}{2} \gamma \sin ^{2} \alpha\right] \\
& \frac{E_{I}(\alpha)}{A}=\gamma+2 \phi\left[\sigma_{S G}(1-\cos \alpha)+\sigma_{S L}(1+\cos \alpha)-\frac{1}{2} \gamma \sin ^{2} \alpha\right]
\end{aligned}
$$

Note that if $\alpha=90^{\circ}$ and the particle surface coverage is maximum $\phi=N_{S} \pi \mathrm{R}^{2} / \mathrm{A} \approx 0.91$, one finds that $E_{I}$ should approach the Antonow's rule for a liquid-solid-gas system $E=\sigma_{S L} A_{P}+\sigma_{S G} A_{P}$, where $A_{P}=N_{S} 2 \pi R^{2}$, see Figure 8f. In general, if particles are in a diluted interfacial regime, and no lateral interactions occur, the interface can be seen as a pure liquid interface populated with isolated particles. Note that standard fluid interfacial tension techniques probe only the interactions mediated by the liquid interface and the presence of isolated non-interacting particles does not affect the interfacial tension $\gamma$ measurements. In fact, pendant drop profile measurements are based on Laplace equation, and $\gamma$ is calculated from the liquid surface curvature. In force measurements, like the Wilhelmy plate technique, $\gamma$ is calculated from the force exerted by the liquid meniscus on the plate. Hence in both techniques, the interfacial tension measured in the presence of isolated non-interacting particles is the one of the pure liquid as reported in several articles [75][77][78][79]. Given the particle irreversible adsorption, a fluid area change does not lead to a fluid interfacial tension change in dilute particle regimes. Note that in presence of particles, the composite interface may show wetting properties related to both roughness (see section 2.1) and inhomogeneity (see section 2.2).

In literature a different description of the interfacial free energy was proposed by Du et al [80],[81]. They write the "total interfacial energy" as:

$E_{I}(\alpha)=\gamma A+N_{S} \Delta E=\gamma A+N_{S}\left[E(\alpha)-E\left(\alpha=0^{\circ}\right.\right.$ or $\left.\left.180^{\circ}\right)\right]$

Note that the previous energy is neither the total energy of the system, Equation 25, nor the free energy of the liquid surface covered by particles, equation 26. The previous equation also predicts that the free energy of the interface depends on the adsorption path of the particles, i.e. in which medium they were first dispersed. Equation 27 was also used to model the effective interfacial tension $\gamma_{\text {eff }}$ measured in pendent drop experiments: $\gamma_{\text {eff }}=E_{I} / A=\gamma+N_{S} \Delta E / A$ [80]. It is important to recall again that the interfacial tension measurement in pendant drop experiments is based on the Laplace equation, which applies strictly for liquid surfaces. Hence, the presence of solid particles, in absence of lateral interactions, is not expected to vary the interactions along the interface and thus should not affect the measurement of the interfacial tension in pendant drop experiments. For nanoparticles, assuming equilibrium between bulk and interface, some other models and interface equations of state have been proposed [82],[83],[84].

Finally, note that the interfacial energy of a liquid surface covered by particles written in equation 26 predicts both a decrease and an increase of the energy. 
Figure 8

\section{Contact line pinning: Hysteresis and Frictions}

Roughness and inhomogeneity of the surface have major impacts not only on the static but also on the dynamic of partial wetting. Contact line pinning occurs whenever a defect is present on the solid surface. Hence the liquid interface deforms around the physical or chemical defect dissipating energy and leading to contact angle hysteresis.

4.1 Deformation of the liquid interface. Even in absence of surface defects the contact line may show some weak distortions, e.g. due to thermal motion. As described by Joanny and de Gennes [6], we consider a distortion of the contact line on the solid along the $\mathrm{x}$-axis, whose displacement is $u(x)$ (at $y=0)$ and its wave vector $q$, see Fig. $9 \mathrm{a}$ and b. As the line is displaced on the solid, also the liquid surface will be perturbed. The distortion along the $x$-axis and the Laplace's equation lead to a liquid surface displacement:

$$
z(x, y)=\alpha y+\frac{1}{2 \pi} \int_{-\infty}^{+\infty} u_{q} \mathrm{e}^{i q x} \mathrm{e}^{-|q| y} d q
$$

Significant distortion of the liquid surface happens over a distance $q^{-1}$ close to the solid comparable to the periodicity of the distortion of the contact line on the solid. The amplitude of the distortion $u_{\mathrm{q}}$ is also fixed by the displacement $u(x)$.

Now we summarize the calculation of the displacement of the contact line assuming an heterogeneous surface, showing weak defect forces that act as random fluctuating forces $f(x)$. In Figure 9b, fluctuations of the contact line are sketched, they follow [6]:

$$
<[u(x)-u(0)]^{2}>=2 D|x|
$$

Where $D=\frac{1}{\gamma^{2} \alpha^{4}} \int_{0}^{+\infty}<f(0) f(x)>d x$. These contact line fluctuations can be significant. The amplitude of the distortion scales as $u^{2} \sim L \xi$, where $\xi$ is the correlation length of the defect and $L$ is the system size [6]. Considering both a planar surface and a solid particle geometries, if $\xi=1 \mathrm{~nm}$ and $L=1 \mu \mathrm{m}$, the amplitude of the contact line displacement is about $30 \mathrm{~nm}$. Note that the latter value is much greater than the amplitude of thermal capillary waves[85] $(\approx 0.2 \mathrm{~nm})$ or the characteristic displacement in the Molecular-kinetic model for friction of partial wetting (0.1-1 nm) [7].

In presence of an isolated defect of size $d$ and force $f_{d}=\gamma d\left[\cos \left(\alpha_{d}\right)-\cos \alpha\right]$, (where $\alpha_{d}$ is the contact angle on the defect, see Figure 9) the contact line on the solid takes the form, see Figure 9c [6]:

$u(x)=\frac{f_{d}}{\pi \gamma \sin ^{2} \alpha} \ln \left(\frac{L}{|x|}\right)$, 
Where $L$ is a macroscopic distance, which could be either the system size or the distance between two adjacent pinning points.

\subsection{Weak or strong pinning}

Weak or strong pinning of the contact line on a single defect may occur depending on the amplitude of the defect force $F_{D}=f_{l}\left(y_{m}-y_{d}\right)$ with respect to the restoring force $F_{R}=K\left(y_{m}-y_{L}\right)$ of the liquid tail, see Figure 9c. $y_{m}$ is the actual position of the line, $y_{d}$ is the defect position, $K=\pi \gamma \sin ^{2} \alpha / \ln (L / d)$ (see equation 30) is the spring constant of the restoring force and $y_{L}$ is the position of the contact line in absence of the defect, see Figure 9c [6],[86].

For weak defects, $f_{l} / K$ is small and no hysteresis of the contact angle is expected. For strong defects, $f_{l} / K$ is large and contact angle hysteresis occurs. The contact line is pinned on the defects and only if enough energy is injected into the system, a line displacement will occur towards a new position. The limiting values of stable macroscopic contact angles are the advancing $\alpha_{A}$ and receding $\alpha_{R}$ contact angles. For a spherical colloid, $\alpha_{A}$ is the stable angle reached after a displacement of the contact line resulting in an increase of the colloid wetted area; whereas $\alpha_{R}$ is the angle reached after a displacement of the contact line resulting in a decrease of the wetted area of the colloid [56], see Figure $9 \mathrm{~d}$ and e.

Figure 9

\subsection{Contact angle Hysteresis}

In the case of strong defects, stable contact angles can be defined by balancing the work $F_{l} \Delta y$ done by the force $F_{l}$ (equation 5 and 6), which tends to move the line towards $\alpha_{e q}$ after a displacement $\Delta y$, and the energy dissipated by the defects. For isolated non-interacting defects [27]:

$F_{l} \Delta y=\phi \Delta y R_{i} \sin \alpha W$

Where $\phi$ is the number of defects per unit area, $\Delta y R_{i} \sin \alpha$ is the area defined by the displacement $\Delta y$, where $R_{i}=R_{0}(\alpha)$ for the drop, and $R_{i}=R$ for the particle geometry respectively. $W$ is the specific energy of a defect, which can be calculated at the maximum elongation $u_{m}=K^{-1} f_{\mathrm{m}}$ (see equation 30) before the line snaps from the defect. Considering the case of isolated defects of size $d$, see Figure 9c [6]:

$$
W=\frac{1}{2} K u_{m}^{2}=\frac{f_{m}^{2} \ln (L / d)}{\gamma \pi \sin ^{2} \alpha} .
$$

In the case of surface roughness, instead, the specific energy $W$ of a topographical defect of size $d$ and height $h$ can be written as [5]: 
$W=\frac{\gamma d^{2} \sin ^{2}\left(\frac{\mathrm{d} h}{\mathrm{~d} y}\right) \ln (L / d)}{2 \pi}$

Where $\frac{\mathrm{d} h}{\mathrm{~d} y}$ is the derivative of the profile height along the y-axis. Assuming that energies dissipated during a contact line advancing and receding steps are the same, one writes the contact angle hysteresis as:

$\cos \alpha_{R}-\cos \alpha_{A}=\phi 2 W$

which increases linearly with the defect concentration $\phi$. For concentrated interacting defects the linear dependency does not hold anymore [87].

Many theoretical and experimental investigations have been carried out for drop contact angle hysteresis on solid substrates in presence of defects of different sizes and structures [6,88-90]. For spherical solid particles at the liquid interface very rarely contact angle hysteresis has been investigated or discussed [22],[56],[91]. Many experimental investigations report on particle contact angle[92] but very few experiments have described distributions of particle contact angles, which we believe are very important to describe line pinning [93],[50].

\subsection{Dynamics and Frictions}

For the planar surface geometry and as well for a particle at a liquid interface, two main sources of dissipations in partial wetting dynamics have been discussed in the literature [94],[7],[95],[21]. We are now concerned with the dynamics of drops or particles occurring during the path from a non-wetting state ( $\alpha=0$ or $180^{\circ}$ ) to an equilibrium position $\left(\alpha_{e q}\right)$. We assume that the velocity $v_{y}$ and the capillary number $\mathrm{Ca}=\eta v_{y} / \gamma$ are small, where $\eta$ is the dynamic viscosity of the liquid. Two main length scales define the sources of dissipations, which are due to the viscous stresses and molecular frictions. For length scales larger than the molecular size ( or $l_{s}$ ), viscous flows and stresses are generated upon the relative motion between a liquid and a solid with a velocity $v_{y}$.

The power dissipated as a function of the contact angle can be written as [96]:

$\Omega=4 g(\alpha) \eta v_{y}^{2} \ln \left(\frac{R_{i}}{a}\right) 2 \pi R_{i} \sin \alpha$

Where $R_{i}=R_{0}(\alpha)$ is the drop radius for the planar surface geometry and $R_{i}=R$ is the particle radius for the particle geometry; $a$ is a molecular size and $g(\alpha)$ is a function of the contact angle: $g(\alpha) \approx 0.756 / \alpha-0.084 \alpha$ [96]. For small contact angles $\alpha<<45^{\circ}, g(\alpha)=3 /(4 \alpha)$ and $\Omega=\frac{3 \eta v_{y}^{2}}{\alpha} \ln \left(\frac{R_{i}}{a}\right) 2 \pi R_{i} \sin \alpha$ [27]. If one writes $\Omega$ as a force $F_{\text {visc }}$ times a velocity, a viscous friction coefficient, $\zeta_{\text {visc }}=F_{\text {visc }} / v_{y}=\Omega / v_{y}{ }^{2}$, can be written as:

$\zeta_{\text {visc }}=4 g(\alpha) \eta \ln \left(\frac{R_{i}}{a}\right) 2 \pi R_{i} \sin \alpha$

Note that this friction does not depend neither on the interfacial tension nor the equilibrium contact angle but it depends only on the viscosity and the geometric contact angle (or dynamic contact angle) experienced during the path from a non-wetting state $\left(\alpha=0\right.$ or $\left.180^{\circ}\right)$ to an equilibrium position $\left(\alpha_{e q}\right)$. It is important to mention that an additional source of viscous dissipation occurs in the presence of a precursor film, which is particularly relevant in complete wetting [97],[5]. To the best of our knowledge, the 
existence of such a precursor film has been never discussed in the complete wetting of solid particles. The existence of such film may occur when hydrophilic particles are deposited on top of a liquid surface in a dry state or using a spreading solvent.

The second source of dissipation is due to molecular frictions. When dealing with length scale comparable to the thermal length $l_{T}=\left[\frac{k_{b} T}{\gamma}\right]^{1 / 2}$, a molecular kinetic theory should be applied to evaluate the dissipation. Thermal agitation energy $k_{b} T$ leads to contact line jumps over distances $\lambda$ occurring at a characteristic time $\tau$. This molecular line friction reads [7,98,99]:

$\zeta_{\text {line }}=\frac{k_{b} T}{\lambda^{3}} \tau 2 \pi R_{i} \sin \alpha$

and $\tau \cong \frac{\eta v_{m}}{k_{b} T} \exp \frac{E_{a}}{k_{b} T}$

Where $v_{m}$ is the molecular volume of the liquid and $E_{a}$ is the activation energy needed for the line jump. Usually $E_{a}$ is written in the form of an adhesion energy $E_{a}=\lambda^{2} \gamma\left(1+\cos \alpha_{e q}\right)$ [7]. However, it is important to remark that the line jump occurs at the molecular level whereas the equilibrium contact angle in the adhesion energy is defined in the macroscopic level. Thus, one may wonder if instead of $\alpha_{e q}$ one should consider the local contact angle which account for long range surface forces selected at a length scale set by $\lambda$, see Figure 3 .

In Figure 10 we plot the viscous and line frictions for drop and particle as a function of $\alpha$. Viscous friction is extremely high for the drop geometry at low contact angles given that the drop radius is very high. If $\alpha$ increases, the friction decreases significantly and it becomes comparable to the Stokes friction $(6 \pi \eta R)$ of a rigid sphere immersed in a liquid. For a particle, the viscous friction is also high at low contact angles but it does not decrease so sharply as for the drop geometry given that the length of the contact line is 0 at $\alpha=$ 0 and maximum at $\alpha=90^{\circ}$. Line friction coefficients depend strongly on $\lambda$ and the activation energy, which depends on $\gamma$ and $\alpha_{e q}$. For a typical values $\lambda=0.6 \mathrm{~nm}$ and $\alpha_{e q}=90^{\circ}$ [7], line frictions can be smaller than the viscous frictions at low contact angles but they become higher than the viscous frictions for $\alpha>20^{\circ}$. For the particle geometry it is important to remark that the highest line friction is reached at $\alpha=90^{\circ}$, where the line length is at maximum.

Now we can briefly discuss the equation of motion of the contact line on the solid for a drop or a particle considering the particular case of driving force plotted in Fig. 2 for $\alpha_{e q}=65^{\circ}$. For a drop starting at $\alpha=$ $180^{\circ}$ the viscous and line friction are low and the force increases if $\alpha$ decreases, hence the line will move towards the equilibrium contact angle. It may approach but never reach the equilibrium in a reasonable experimental time, given that the force decreases and the frictions increases when $\alpha$ gets close to $\alpha_{e q}$. For a particle starting at $\alpha_{e q}=180^{\circ}$ the motion may slow down well before $\alpha_{e q}$ because line friction is maximum at $\alpha=90^{\circ}$. Also for a particle starting at $\alpha=0^{\circ}$ it is important to remark that the driving force is low and the viscous friction is very high at low contact angles.

Partial wetting dynamics for drops have been largely explored theoretically and experimentally and combined viscous and line friction models have been also used [100,101],[5,27]. On the contrary, very few experiments have been carried out for the dynamics of particle at the liquid interface. The slow dynamics of a particle breaching an oil-water interface have been recently investigated by Kaz et al. [21]. 
They indeed observed that particles either are not able to breach the interface or they move very slowly at the interface. These results agree with our discussion before, see Fig. 10. Dynamics of dewetting is also largely reported in the literature for liquids on solid substrates [102,103]. Nucleation of dry region on the solid surface may occur due to surface defects or by the amplification of capillary waves [104]. Also in this case, we are not aware of experimental studies on details of dewetting mechanisms for particles at the liquid interface.

Figure 10

Finally, viscous and line frictions do not affect only the contact line motion during a partial wetting dynamic towards equilibrium. For particles straddling an interface at a constant contact angle, even if the contact angle does not vary, the contact line may fluctuate and a line friction could manifest. Recent experiments on particle lateral and the rotational motions show the strong influence of the viscous and line frictions due to contact line fluctuations, which decrease the particle diffusion coefficients $[23,105],[56]$.

\section{Conclusions and Outlook}

In this review, we describe fundamental aspects of the partial wetting for drops and particles. Even if theoretical and experimental works have been extensively done, many new investigations deserve consideration. For smooth surfaces, line tension could affect the wetting of drop or particles of small sizes in a very different way (section 1.2). New experiments for nanodrops and for particles of different aspect ratios (ellipsoids or disks) and contact angles could be designed in order to address line tension effects. It would be also interesting to gain some control on the sign and value of the line tension by using lineactive molecules able to tune line tension in the partial wetting of drops and particles. For particles, the impact of surface roughness could be severe. Note that not only the contact angle may shift, but also that the adsorption energy minima $\Delta E=E(\alpha)-E\left(\alpha=0^{\circ}\right.$ or $\left.180^{\circ}\right)$ could reduce or even vanish. Patchy and Janus particles could be used for experimental investigations in order to elucidate on the applications of the Cassie-Baxter equation (Section 2). Some open questions remain on the information provided by the effective interfacial tension measure in experiments for liquid covered by particles (Section 3). Finally, new experiments on contact angle distributions and dynamics of particles at the water surface may provide new insights into the physics of contact line pinning and line frictions, that can be compared to the results obtained for drops (see Section 4).

\section{ACKNOLDEGEMENTS}

We thank Martin In and Dario Donnarumma for discussions and for helping with the Graphical Abstract. Financial supports from Labex Chemisyst, Labex Numev and ANR SURFANICOL ANR-14-CE07-003901 are also acknowledged. 


\section{REFERENCES}

[1] Sedev R. The molecular-kinetic approach to wetting dynamics: Achievements and limitations. Adv Colloid Interface Sci 2014;222:661-9. doi:10.1016/j.cis.2014.09.008.

[2] Mugele F, Baret J-C. Electrowetting: from basics to applications. J Phys Condens Matter 2005;17:R705-74. doi:10.1088/0953-8984/17/28/R01.

[3] Lipowsky R. The conformation of membranes. Nature 1991;349. doi:10.1038/349475a0.

[4] Thiam AR, Farese R V, Walther TC. The biophysics and cell biology of lipid droplets. Nat Rev Mol Cell Biol 2013;14:775-86. doi:10.1038/nrm3699.

[5] De Gennes PG. Wetting: Statics and dynamics. Rev Mod Phys 1985;57:827-63. doi:10.1103/RevModPhys.57.827.

[6] Joanny JF, De Gennes PG. A model for contact angle hysteresis. J Chem Phys 1984;81:552. doi:10.1063/1.447337.

[7] Blake TD. The physics of moving wetting lines. J Colloid Interface Sci 2006;299:1-13. doi:10.1016/j.jcis.2006.03.051.

[8] Ralston J, Fornasiero D, Hayes R. Bubble - particle attachment and detachment in flotation. Int J Miner Process 1999;56:133-64.

[9] Vignati E, Piazza R, Lockhart TP. Pickering Emulsions: Interfacial Tension, Colloidal Layer Morphology, and Trapped-Particle Motion. Langmuir 2003;19:6650-6. doi:10.1021/la034264l.

[10] Stocco A, Rio E, Binks BP, Langevin D. Aqueous foams stabilized solely by particles. Soft Matter 2011;7:1260. doi:10.1039/c0sm01290d.

[11] Stocco A, Chanana M, Su G, Cernoch P, Binks BP, Wang D. Bidirectional nanoparticle crossing of oil-water interfaces induced by different stimuli: insight into phase transfer. Angew Chemie Int Ed 2012;51:9647-51. doi:10.1002/anie.201203493.

[12] Geisel K, Isa L, Richtering W. Unraveling the 3D localization and deformation of responsive microgels at oil/water interfaces: a step forward in understanding soft emulsion stabilizers. Langmuir 2012;28:15770-6. doi:10.1021/la302974j.

[13] Faraudo J, Bresme F. Interplay between thermodynamics and geometry in the adsorption of nonspherical nanoparticles at liquid/liquid interfaces. J Non-Equilibrium Thermodyn 2004;29:397404. doi:10.1515/JNETDY.2004.064.

[14] Lin Y, Böker A, Skaff H, Cookson D, Dinsmore a D, Emrick T, et al. Nanoparticle assembly at fluid interfaces: structure and dynamics. Langmuir 2005;21:191-4. doi:10.1021/1a048000q.

[15] Kaptay G. Interfacial criteria for stabilization of liquid foams by solid particles. Colloids Surfaces A Physicochem Eng Asp 2003;230:67-80. doi:10.1016/j.colsurfa.2003.09.016.

[16] Nonomura Y, Komura S, Tsujii K. Adsorption of Microstructured Particles at Liquid - Liquid Interfaces 2006:13124-9.

[17] Wang D, Yordanov S, Paroor HM, Mukhopadhyay A, Li CY, Butt H-J, et al. Probing diffusion of single nanoparticles at water-oil interfaces. Small 2011;7:3502-7. doi:10.1002/smll.201101823.

[18] Drelich J. The significance and magnitude of the line tension in three-phase (solid-liquid-fluid) systems. Colloids Surfaces A Physicochem Eng Asp 1996;116:43-54. doi:10.1016/09277757(96)03651-5. 
[19] Maestro A, Bonales LJ, Ritacco H, Rubio RG, Ortega F. Effect of the spreading solvent on the three-phase contact angle of microparticles attached at fluid interfaces. Phys Chem Chem Phys 2010;12:14115-20. doi:10.1039/c0cp00570c.

[20] De Graaf J, Dijkstra M, van Roij R. Adsorption trajectories and free-energy separatrices for colloidal particles in contact with a liquid-liquid interface. J Chem Phys 2010;132:164902. doi:10.1063/1.3389481.

[21] Kaz DM, Mcgorty R, Mani M, Brenner MP, Manoharan VN. Physical ageing of the contact line on colloidal particles at liquid interfaces. Nat Mater 2012;11:138-42. doi:10.1038/nmat3190.

[22] Preuss M, Butt H. Measuring the Contact Angle of Individual Colloidal Particles. J Colloid Interface Sci 1998;208:468-77. doi:10.1006/jcis.1998.5833.

[23] Boniello G, Blanc C, Fedorenko D, Medfai M, Mbarek N Ben, In M, et al. Brownian diffusion of a partially wetted colloid. Nat Mater 2015;14:908-11. doi:10.1038/nmat4348.

[24] Du K, Liddle JA, Berglund AJ. Three-dimensional real-time tracking of nanoparticles at an oilwater interface. Langmuir 2012;28:9181-8. doi:10.1021/la300292r.

[25] De Gennes PG, Hua X, Levinson P. Dynamics of wetting: local contact angles. J Fluid Mech 1990;212:55-63. doi:10.1017/S0022112090001859.

[26] Kaptay G. Interfacial Forces in Dispersion Science and Technology. J Dispers Sci Technol 2012;33:130-40. doi:10.1080/01932691.2010.548232.

[27] De Gennes P-G, Brochard-Wyart F, Quéré D. Capillarity and Wetting Phenomena. 2004. doi:10.1007/978-0-387-21656-0.

[28] Sharma A. Equilibrium contact angles and film thicknesses in the apolar and polar systems: Role of intermolecular interactions in coexistence of drops with thin films. Langmuir 1993:3580-6. doi:10.1021/la00036a038.

[29] Pieranski P. Two-Dimensional Interfacial colloidal crystal. Phys Rev Lett 1980;45:569.

[30] Stocco A, Möhwald H. The influence of long-range surface forces on the contact angle of nanometric droplets and bubbles. Langmuir 2015;31:11835-41. doi:10.1021/acs.langmuir.5b02922.

[31] Schimmele L, Naplórkowski M, Dietrich S. Conceptual aspects of line tensions. J Chem Phys 2007;127:164715. doi:10.1063/1.2799990.

[32] Drelich J. The significance and magnitude of the line tension in three-phase (solid-liquid-fluid) systems. Colloids Surfaces A Physicochem Eng Asp 1996;116:43-54. doi:10.1016/09277757(96)03651-5.

[33] Tadmor R. Line energy and the relation between advancing, receding, and young contact angles. Langmuir 2004;20:7659-64. doi:10.1021/la049410h.

[34] Aveyard R, Clint JH. Particle wettability and line tension. J Chem Soc Faraday Trans 1996;92:85. doi:10.1039/ft9969200085.

[35] Berg JK, Weber CM, Riegler H. Impact of Negative Line Tension on the Shape of Nanometer-Size Sessile Droplets. Phys Rev Lett 2010;105:76103. doi:10.1103/PhysRevLett.105.076103.

[36] Checco A, Guenoun P, Daillant J. Nonlinear dependence of the contact angle of nanodroplets on contact line curvature. Phys Rev Lett 2003;91:186101. doi:10.1103/PhysRevLett.91.186101.

[37] Checco A, Schollmeyer H, J.Daillant, Guenoun P, Boukherroub R. Nanoscale wettability of selfassembled monolayers investigated by noncontact atomic force microscopy Investigated by Noncontact Atomic Force Microscopy. Languir 2006;22:116-26. 
[38] Honerkamp-Smith AR, Cicuta P, Collins MD, Veatch SL, den Nijs M, Schick M, et al. Line tensions, correlation lengths, and critical exponents in lipid membranes near critical points. Biophys J 2008;95:236-46. doi:10.1529/biophysj.107.128421.

[39] García-Sáez AJ, Chiantia S, Schwille P. Effect of line tension on the lateral organization of lipid membranes. J Biol Chem 2007;282:33537-44. doi:10.1074/jbc.M706162200.

[40] Seemann R, Herminghaus S, Jacobs K. Gaining control of pattern formation of dewetting liquid films. J Phys Condens Matter 2001;13:4925-38. doi:10.1088/0953-8984/13/21/319.

[41] Pompe T, Herminghaus S. Three-Phase Contact Line Energetics from Nanoscale Liquid Surface Topographies. Phys Rev Lett 2000;85:1930-3. doi:10.1103/PhysRevLett.85.1930.

[42] Seemann R, Jacobs K, Blossey R. Polystyrene nanodroplets. J Phys-Condes Matter 2001;13:491523. doi:10.1088/0953-8984/13/21/318.

[43] Delabre U, Cazabat AM. Coalescence driven by line tension in thin nematic films. Phys Rev Lett 2010;104:2-5. doi:10.1103/PhysRevLett.104.227801.

[44] Dehghan A, Pastor KA, Shi AC. Line tension of multicomponent bilayer membranes. Phys Rev E Stat Nonlinear, Soft Matter Phys 2015;91:1-8. doi:10.1103/PhysRevE.91.022713.

[45] Dong L, Johnson DT. Adsorption of acicular particles at liquid-fluid interfaces and the influence of the line tension. Langmuir 2005;21:3838-49. doi:10.1021/la047851v.

[46] Getta T, Dietrich S. Line tension between fluid phases and a substrate. Phys Rev E 1998;57:65571. doi:10.1103/PhysRevE.57.655.

[47] Amirfazli A, Neumann AW. Status of the three-phase line tension: A review. Adv Colloid Interface Sci 2004;110:121-41. doi:10.1016/j.cis.2004.05.001.

[48] Lohse D, Zhang X. Surface nanobubbles and nanodroplets. Rev Mod Phys 2015;87:981-1035. doi:10.1103/RevModPhys.87.981.

[49] McBride SP, Law BM. Influence of line tension on spherical colloidal particles at liquid-vapor interfaces. Phys Rev Lett 2012;109:1-5. doi:10.1103/PhysRevLett.109.196101.

[50] Isa L, Lucas F, Wepf R, Reimhult E. Measuring single-nanoparticle wetting properties by freezefracture shadow-casting cryo-scanning electron microscopy. Nat Commun 2011;2:438. doi:10.1038/ncomms1441.

[51] Churaev N., Starov V., Derjaguin B. The shape of the transition zone between a thin film and bulk liquid and the line tension. J Colloid Interface Sci 1982;89:16-24. doi:10.1016/00219797(82)90115-1.

[52] Wayner P. The Interfacial Profile in the Contact Line Region and the Young-Dupr6 Equation. J Colloid Interface Sci 1982;88:6-7.

[53] Wayner P. Interfacial profile in the contact line region of a finite contact angle system. J Colloid Interface Sci 1980;77:495-500. doi:10.1016/0021-9797(80)90322-7.

[54] A sign minus is missing in the disjoining pressure equation in [30]. This mistake does not affect the results of the article. However, a correction is needed because the terms "attractive" and "repulsive" should be interchanged. n.d.

[55] Wenzel RN. Resistance of solid surfaces to wetting by water. J Ind Eng Chem (Washington, D C) 1936;28:988-94. doi:10.1021/ie50320a024.

[56] Wang X, In M, Blanc C, Malgaretti P, Nobili M, Stocco A. Wetting and orientation of catalytic Janus colloids at the surface of water. Faraday Discuss 2016;191:305-24.

doi:10.1039/C6FD00025H. 
[57] San-Miguel A, Behrens SH. Influence of nanoscale particle roughness on the stability of pickering emulsions. Langmuir 2012;28:12038-43. doi:10.1021/la302224v.

[58] Lenz P, Lipowsky R. Morphological Transitions of Wetting Layers on Structured Surfaces. Phys Rev Lett 1998;80:1920-3. doi:10.1103/PhysRevLett.80.1920.

[59] Blecua P, Lipowsky R, Kierfeld J. Line Tension Effects for Liquid Droplets on Circular Surface Domains 2006:11041-59.

[60] Extrand CW. Contact angles and hysteresis on surfaces with chemically heterogeneous islands. Langmuir 2003;19:3793-6. doi:10.1021/la0268350.

[61] Milne a JB, Amirfazli a. The Cassie equation: how it is meant to be used. Adv Colloid Interface Sci 2012;170:48-55. doi:10.1016/j.cis.2011.12.001.

[62] Liu TL, Chen Z, Kim C-J. A dynamic Cassie-Baxter model. Soft Matter 2015;11:1589-96. doi:10.1039/C4SM02651A.

[63] Kwon Y, Choi S, Anantharaju N, Lee J, Panchagnula M V., Patankar NA. Is the Cassie-Baxter formula relevant? Langmuir 2010;26:17528-31. doi:10.1021/la102981e.

[64] Larsen ST, Taboryski R. A cassie-like law using triple phase boundary line fractions for faceted droplets on chemically heterogeneous surfaces. Langmuir 2009;25:1282-4.

doi:10.1021/la8030045.

[65] Gao L, McCarthy TJ. How Wenzel and Cassie were wrong. Langmuir 2007;23:3762-5. doi:10.1021/la062634a.

[66] Marmur A, Bittoun E. When Wenzel and Cassie are right: reconciling local and global considerations. Langmuir 2009;25:1277-81. doi:10.1021/la802667b.

[67] Casagrande C, Fabre P, Raphaël E, Veyssié M. "Janus Beads": Realization and Behaviour at Water/Oil Interfaces. Europhys Lett 2007;9:251-5. doi:10.1209/0295-5075/9/3/011.

[68] Ondarçuhu T, Fabre P, Raphaël E, Veyssié M. Specific properties of amphiphilic particles at fluid interfaces. J Phys 1990;51:1527-36. doi:10.1051/jphys:0199000510140152700.

[69] Aveyard R. Can Janus particles give thermodynamically stable Pickering emulsions? Soft Matter 2012;8:5233. doi:10.1039/c2sm07230k.

[70] Rezvantalab H, Shojaei-Zadeh S. Capillary interactions between spherical Janus particles at liquidfluid interfaces. Soft Matter 2013;9:3640. doi:10.1039/c3sm27380f.

[71] Wang X, In M, Blanc C, Malgaretti P, Nobili M. Wetting and orientation of catalytic Janus colloids at the surface of water n.d.

[72] Bonn D, Eggers J, Indekeu J, Meunier J. Wetting and spreading. Rev Mod Phys 2009;81:739-805. doi:10.1103/RevModPhys.81.739.

[73] Okubo T. Suface Tension of Structured Colloidal Suspensions of Polystyrene and Silica Spheres at the Air-Water Interface. J Colloid Interface Sci 1995;171:55-62. doi:http://dx.doi.org/10.1006/jcis.1995.1150.

[74] Binks BP. Particles as surfactants - Similarities and differences. Curr Opin Colloid Interface Sci 2002;7:21-41. doi:10.1016/S1359-0294(02)00008-0.

[75] Aveyard R, Clint JH, Nees D, Paunov VN. Compression and Structure of Monolayers of Charged Latex Particles at Air / Water and Octane / Water Interfaces. Langmuir 2000:1969-79.

[76] Oettel M, Dietrich S. Colloidal interactions at fluid interfaces. Langmuir 2008;24:1425-41. doi:10.1021/la702794d.

[77] Truzzolillo D, Sharaf H, Jonas U, Loppinet B, Vlassopoulos D. Tuning the Structure and Rheology 
of Polystyrene Particles at the Air-Water Interface by Varying the pH. Langmuir 2016;32:6956-66. doi:10.1021/acs.langmuir.6b01969.

[78] Zang D, Stocco A, Langevin D, Wei B, Binks BP. An ellipsometry study of silica nanoparticle layers at the water surface. Phys Chem Chem Phys 2009;11:9522-9. doi:10.1039/b907903c.

[79] Bykov AG, Noskov BA, Loglio G, Lyadinskaya V V., Miller R. Dilational surface elasticity of spread monolayers of polystyrene microparticles. Soft Matter 2014;10:6499. doi:10.1039/C4SM00782D.

[80] Du K, Glogowski E, Emrick T, Russell TP, Dinsmore AD. Adsorption energy of nano- and microparticles at liquid-liquid interfaces. Langmuir 2010;26:12518-22. doi:10.1021/la100497h.

[81] Bizmark N, Ioannidis MA, Henneke DE. Irreversible adsorption-driven assembly of nanoparticles at fluid interfaces revealed by a dynamic surface tension probe. Langmuir 2014;30:710-7. doi:10.1021/la404357j.

[82] Wi HS, Cingarapu S, Klabunde KJ, Law BM. Nanoparticle adsorption at liquid-vapor surfaces: Influence of nanoparticle thermodynamics, wettability, and line tension. Langmuir 2011;27:997984. doi:10.1021/la201791g.

[83] Groot RD, Stoyanov SD. Equation of state of surface-adsorbing colloids. Soft Matter 2010;6:1682. doi:10.1039/b925353j.

[84] Fan H, Striolo A. Nanoparticle effects on the water-oil interfacial tension. Phys Rev E 2012;86:51610. doi:10.1103/PhysRevE.86.051610.

[85] Bonn D, Wegdam G. Capillary waves and ellipsometry experiments. J Phys 1992;2:1755.

[86] Ramos S, Tanguy a. Pinning-depinning of the contact line on nanorough surfaces. Eur Phys J E Soft Matter 2006;19:433-40. doi:10.1140/epje/i2005-10056-0.

[87] Ramiasa M, Ralston J, Fetzer R, Sedev R. The influence of topography on dynamic wetting. Adv Colloid Interface Sci 2014;206:275-93. doi:10.1016/j.cis.2013.04.005.

[88] Giacomello A, Schimmele L, Dietrich S. Wetting hysteresis induced by nanodefects. Proc Natl Acad Sci 2015:201513942. doi:10.1073/pnas.1513942113.

[89] Reyssat M, Quéré D. Contact Angle hysteresis generated by strong dilute defects. J Phys Chem B 2009;113:3906-9. doi:10.1021/jp8066876.

[90] Meglio J-M Di, Quéré D. Contact Angle Hysteresis: a First Analysis of the Noise of the Creeping Motion of the Contact Line. Europhys Lett 2007;11:163-8. doi:10.1209/0295-5075/11/2/012.

[91] Stocco A, Su G, Nobili M, In M, Wang D. In situ assessment of the contact angles of nanoparticles adsorbed at fluid interfaces by multiple angle of incidence ellipsometry. Soft Matter 2014:69997007. doi:10.1039/c4sm00482e.

[92] Maestro A, Guzmán E, Ortega F, Rubio RG. Contact angle of micro- and nanoparticles at fluid interfaces. Curr Opin Colloid Interface Sci 2014;19:355-67. doi:10.1016/j.cocis.2014.04.008.

[93] Snoeyink C, Barman S, Christopher GF. Contact angle distribution of particles at fluid interfaces. Langmuir 2015;31:891-7. doi:10.1021/la5040195.

[94] Brochard-Wyart F, De Gennes PG. Dyanmics of Partial Wetting. Adv Colloid Interface Sci 1992;39:1.

[95] Snoeijer JH, Andreotti B. Moving Contact Lines: Scales, Regimes, and Dynamical Transitions. Annu Rev Fluid Mech 2013;45:269-92. doi:10.1146/annurev-fluid-011212-140734.

[96] Kim H-Y, Lee HJ, Kang BH. Sliding of liquid drops down an inclined solid surface. J Colloid Interface Sci 2002;247:372-80. doi:10.1006/jcis.2001.8156. 
[97] Popescu MN, Oshanin G, Dietrich S, Cazabat AM. Precursor films in wetting phenomena. J Phys Condens Matter 2012;24:243102. doi:10.1088/0953-8984/24/24/243102.

[98] Blake TD, Shikhmurzaev YD. Dynamic wetting by liquids of different viscosity. J Colloid Interface Sci 2002;253:196-202. doi:10.1006/jcis.2002.8513.

[99] Duvivier D, Seveno D, Rioboo R, Blake TD, De Coninck J. Experimental evidence of the role of viscosity in the molecular kinetic theory of dynamic wetting. Langmuir 2011;27:13015-21. doi:10.1021/la202836q.

[100] Petrov P, Petrov I. A combined molecular-hydrodynamic approach to wetting kinetics. Langmuir 1992;8:1762-7. doi:10.1021/la00043a013.

[101] De Ruijter MJ, De Coninck J, Oshanin G. Droplet spreading: Partial wetting regime revisited. Langmuir 1999;15:2209-16. doi:10.1021/la971301y.

[102] Redon C, Brochard-Wyart F, Rondelez F. Dynamics of dewetting. Phys Rev Lett 1991;66:715-8. doi:10.1103/PhysRevLett.66.715.

[103] Bertrand E, Blake TD, De Coninck J. Dynamics of dewetting. Colloids Surfaces A Physicochem Eng Asp 2010;369:141-7. doi:10.1016/j.colsurfa.2010.08.006.

[104] Seemann R, Herminghaus S, Jacobs K. Dewetting patterns and molecular forces: A reconciliation. Phys Rev Lett 2001;86:5534-7. doi:10.1103/PhysRevLett.86.5534.

[105] Boniello G, Stocco A, Gross M, In M, Blanc C, Nobili M. Translational viscous drags of an ellipsoid straddling an interface between two fluids. Phys Rev E 2016;94:12602. doi:10.1103/PhysRevE.94.012602. 
a) Liquid drop on planar solid

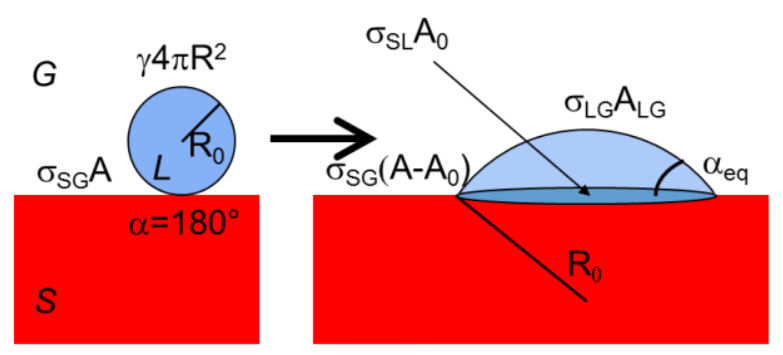

b) Solid particle on liquid surface

vs.

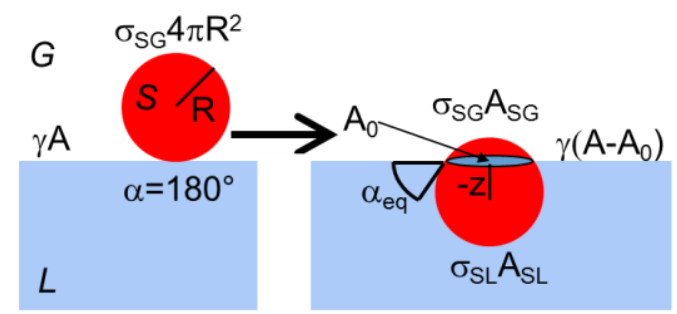

Figure 1 Sketch of a liquid drop on a solid substrate (a) and a solid spherical particle on a liquid interface (b) reaching an equilibrium contact angle in partial wetting from a dry state, $\alpha=180^{\circ}$. $G=$ gas, $S=$ solid, $L=$ liquid.
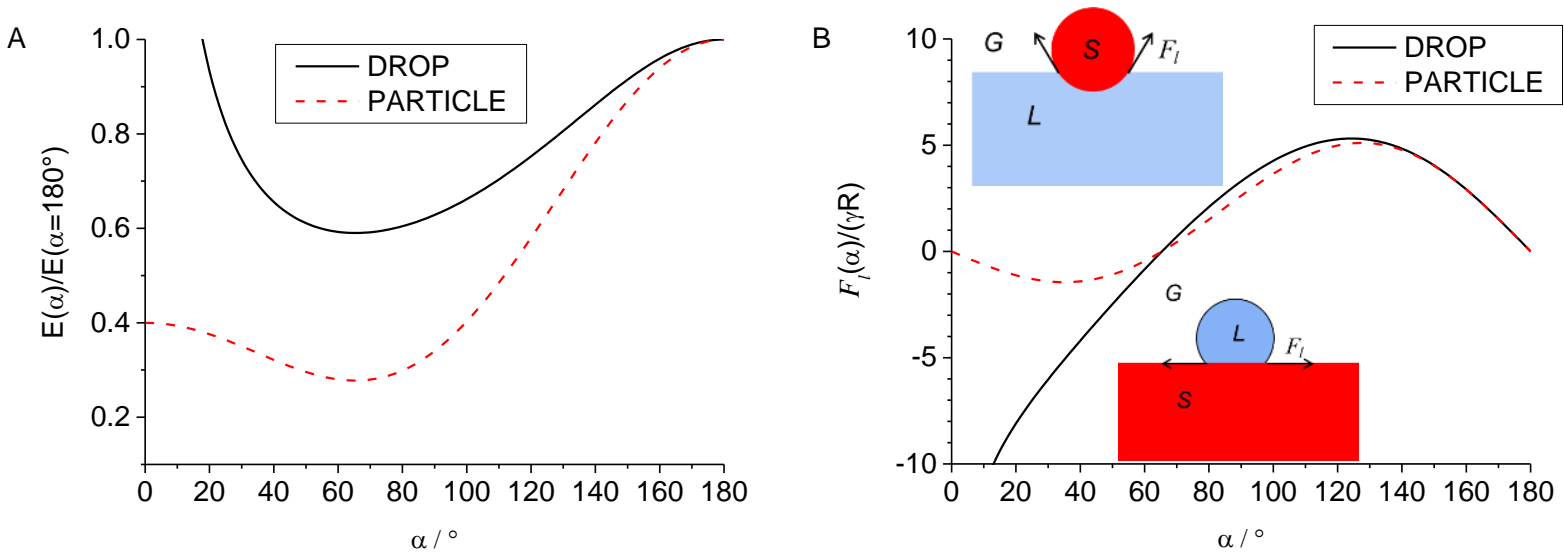

Figure 2 A) Free energy ratio $E(\alpha) / E\left(\alpha=180^{\circ}\right)\left(\alpha_{e q}=65^{\circ}\right.$ and $\gamma=72 \mathrm{mN} / \mathrm{m}, \gamma A$ is a constant of the system and its contribution was subtracted to $E$ ) as a function of $\alpha$ for drop and particle geometries. B) Force $F_{l}$ normalized by the constant term $\gamma R\left(\alpha_{e q}=65^{\circ}, \gamma=72 \mathrm{mN} / \mathrm{m}\right.$ and $\left.R=R_{0}\left(\alpha=180^{\circ}\right)\right)$ as a function of $\alpha$ for drop and particle geometries. 


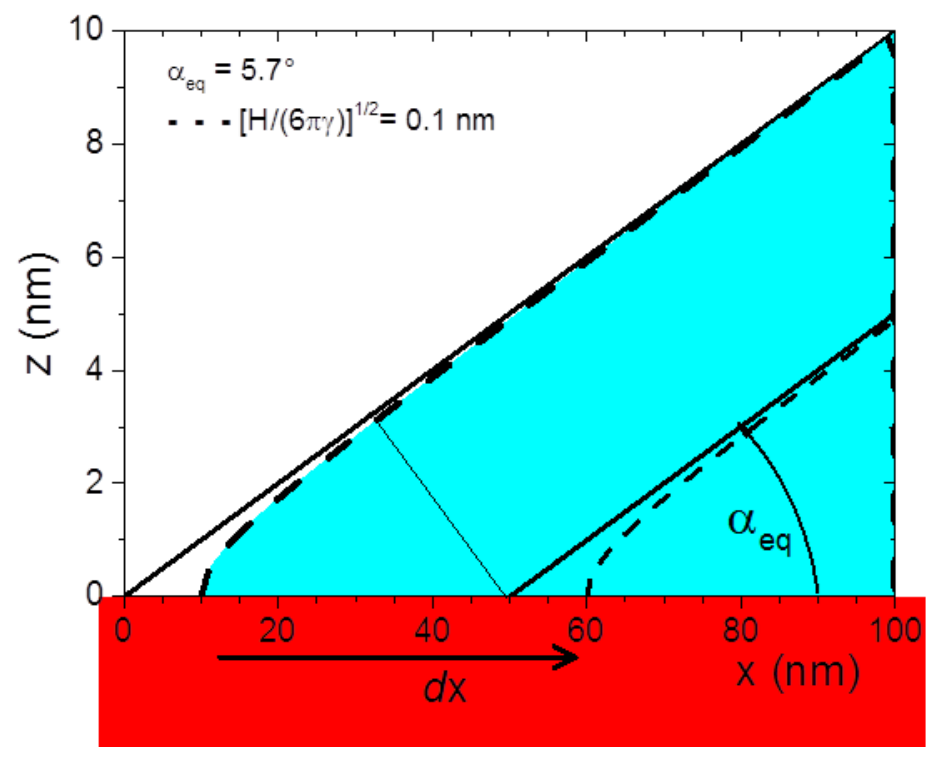

Figure 3 Local contact line profile for a droplet showing attractive VdW long range forces [30].
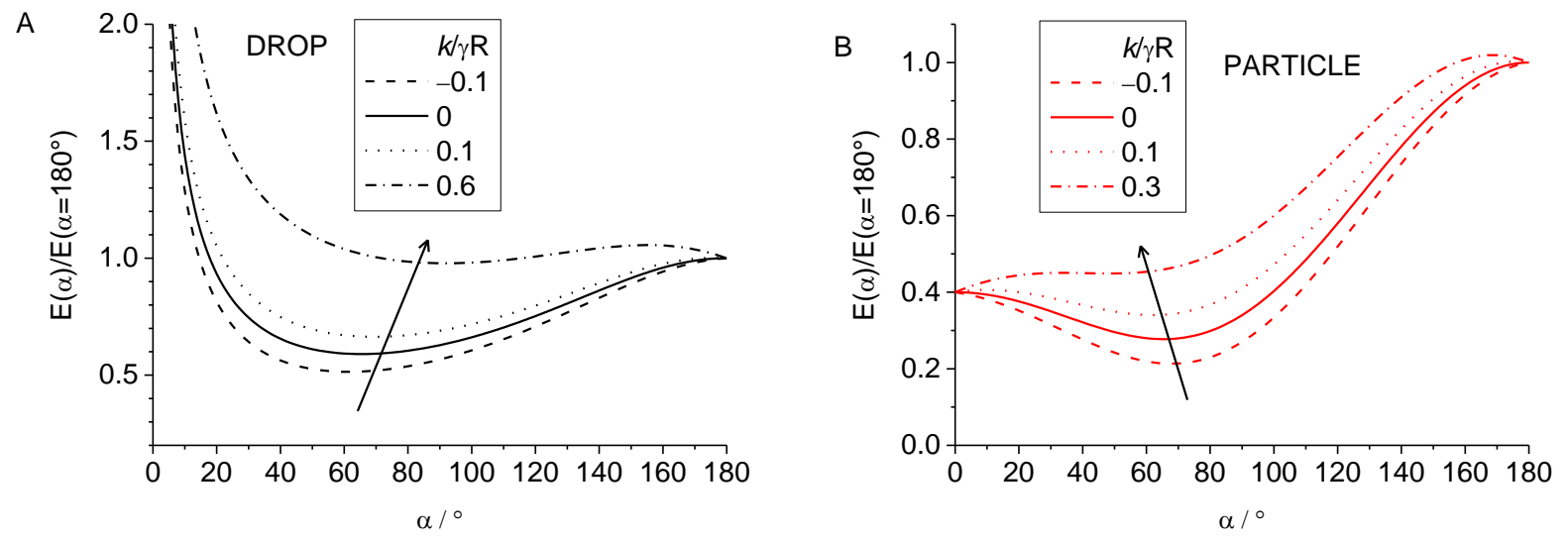

Figure 4 A) Free energy ratio $E(\alpha) / E\left(\alpha=180^{\circ}\right)\left(\alpha_{e q}=65^{\circ}\right.$ and $\gamma=72 \mathrm{mN} / \mathrm{m}, \gamma \mathrm{A}$ is a constant of the system and its contribution was subtracted to $E$ ) as a function of $\alpha$ for the drop geometry and different line tension values. B) Free energy ratios $E(\alpha) / E\left(\alpha=180^{\circ}\right) \quad\left(\alpha_{e q}=65^{\circ}\right.$ and $\gamma=72 \mathrm{mN} / \mathrm{m}, \gamma A$ is a constant of the system and was subtracted to $\left.E\right)$ as a function of $\alpha$ for the particle geometry and different line tension values. 


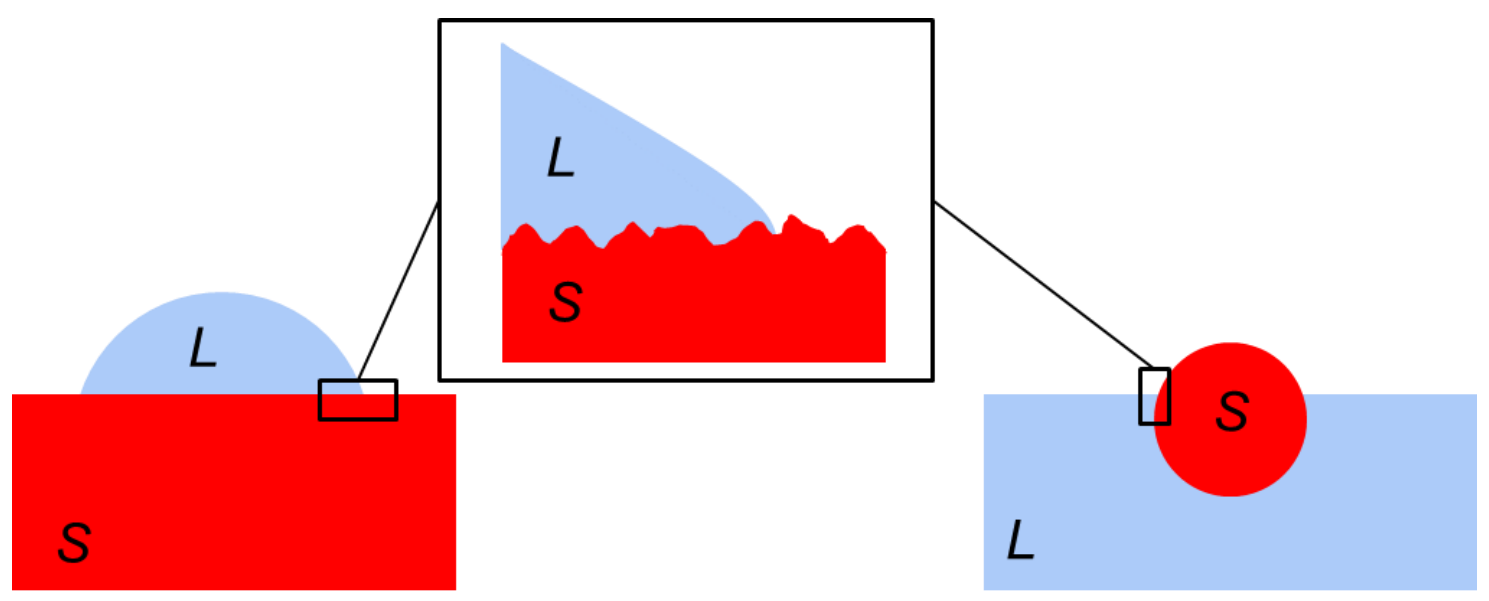

Figure 5 Wenzel model for drop and particle geometries.
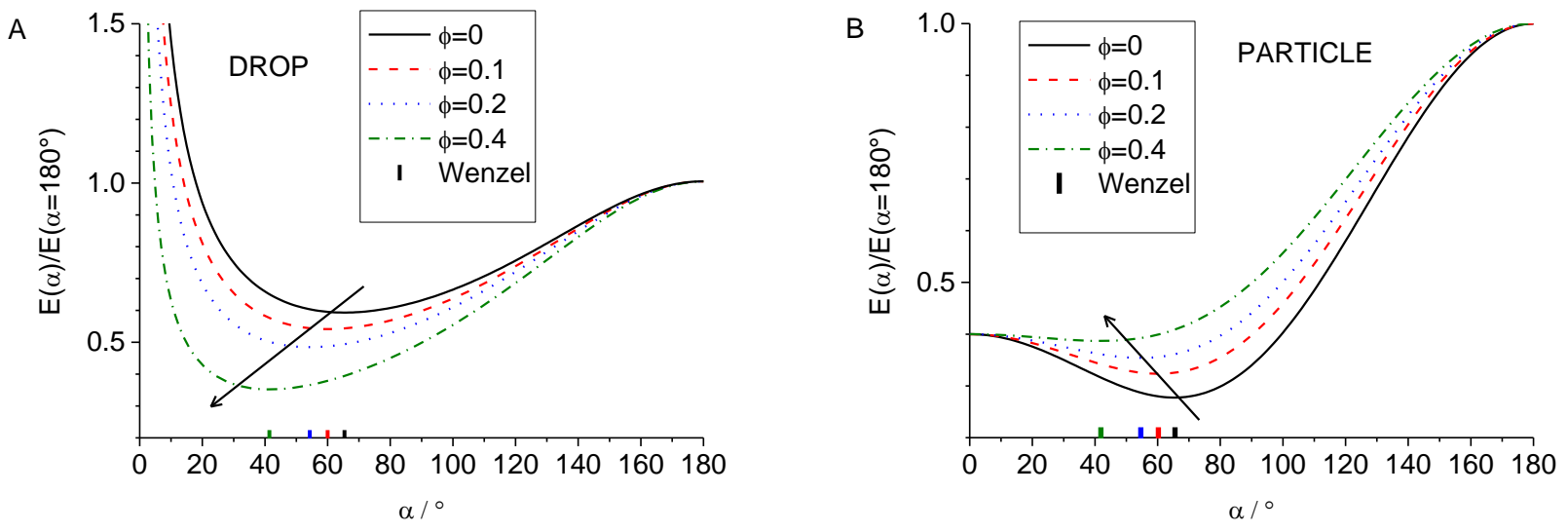

Figure 6 A) Free energy ratio $E(\alpha) / E\left(\alpha=180^{\circ}\right) \quad\left(\alpha_{e q}=65^{\circ}\right.$ and $\gamma=72 \mathrm{mN} / \mathrm{m}, h_{s} / R_{s}=1, \gamma \mathrm{A}$ is a constant of the system and its contribution was subtracted to $E$ ) as a function of $\alpha$ for the drop geometry and different defect concentrations $\phi_{s}$. B) Free energy ratio $E(\alpha) / E\left(\alpha=180^{\circ}\right) \quad\left(\alpha_{e q}=65^{\circ}\right.$ and $\gamma=72 \mathrm{mN} / \mathrm{m}, h_{s} / R_{s}=1, \gamma A$ is a constant of the system and its contribution was subtracted to $E$ ) as a function of $\alpha$ for the particle geometry and different defect concentrations $\phi_{s}$. In the $x$-axis, data points show the equilibrium contact angles calculated using the the Wenzel equation. 


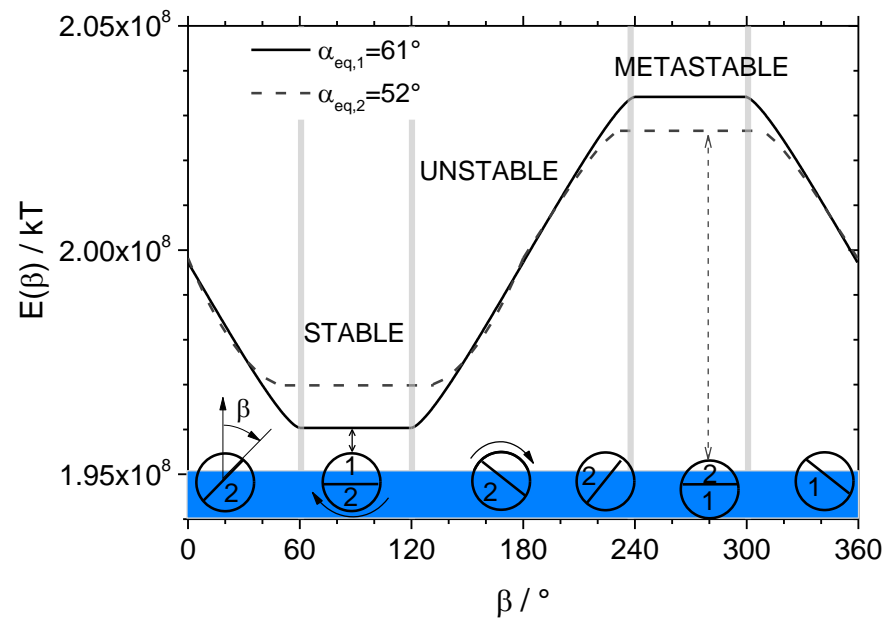

Figure 7 Free energy ( $\gamma=72 \mathrm{mN} / \mathrm{m}, R=1 \mu \mathrm{m}, \gamma \mathrm{A}$ is a constant of the system and its contribution was subtracted to $E$ ) of a Janus particle as a function of the orientation $\beta$ (defined in the left bottom corner) for two different position corresponding to the equilibrium contact angles of each face of the Janus particle [71]. 
a)

S

b)
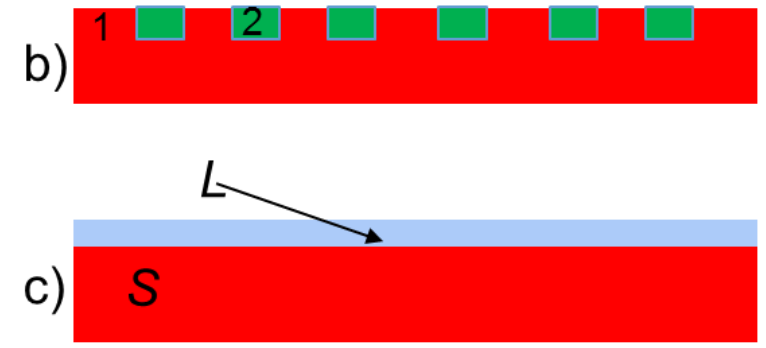

d) $L$

e)

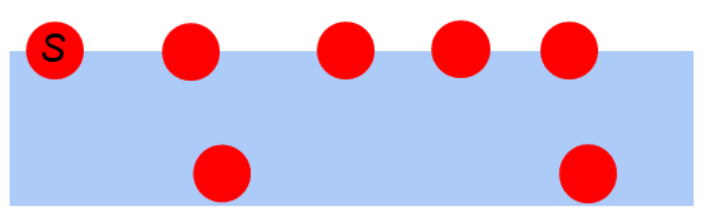

f)

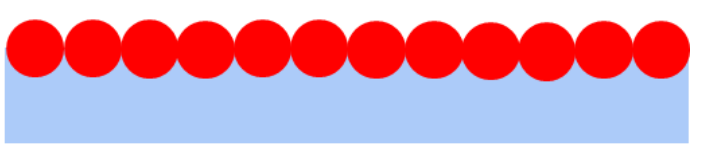

Figure 8 Sketches of solid planar surfaces $(a, b, c)$ and liquid interfaces $(d, e, f)$ populated by a different material as discrete particles (b, e) or films (c, f). 

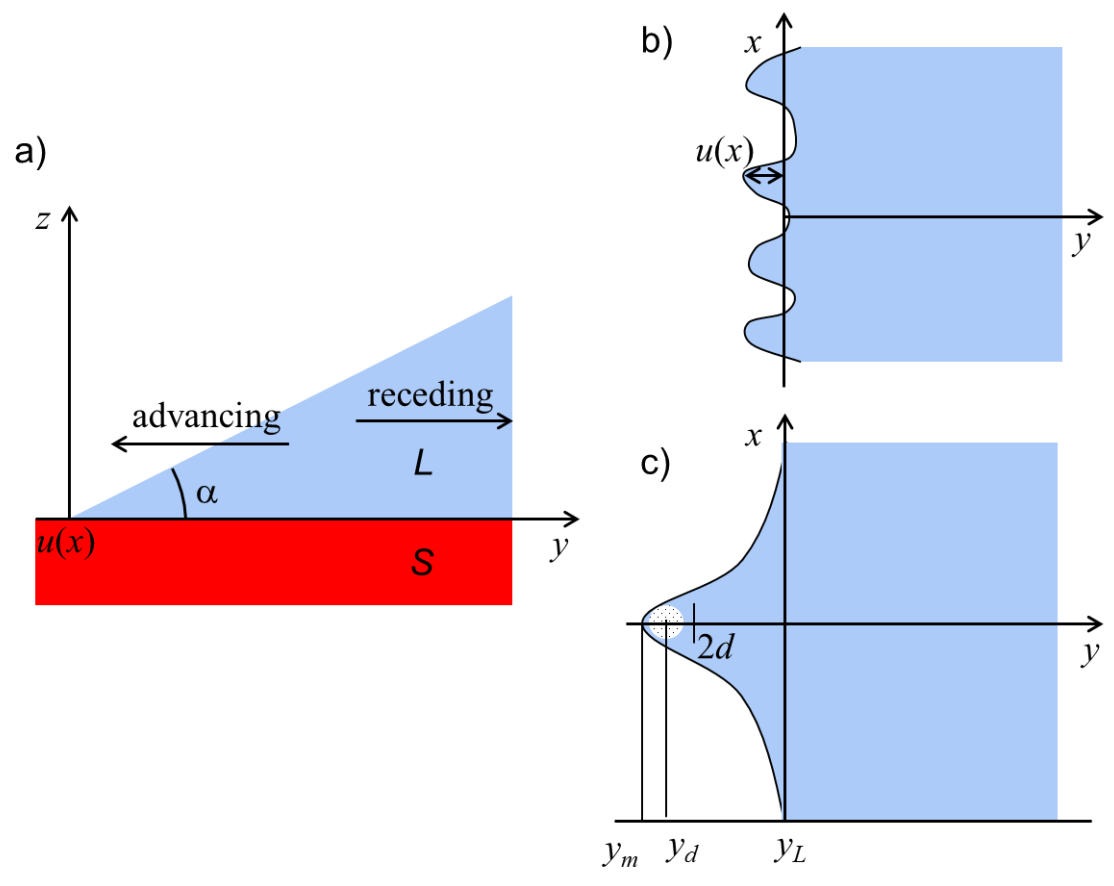

d)

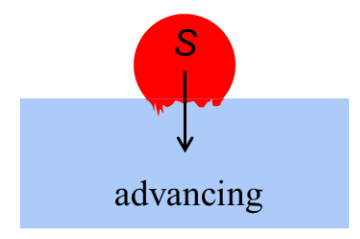

e)

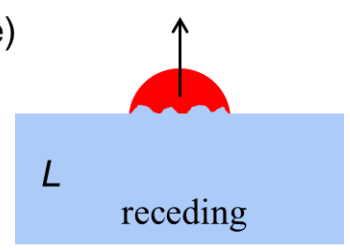

Figure 9 a) Side view of a drop wedge at a given contact angle $\alpha$ and directions of advancing and receding contact lines. b) Top view of the contact line region in absence of strong defects. c) Top view of the contact line region in presence of a strong defect inducing line pinning. d) Side view of a solid particle immerging in a liquid with an advancing contact line. e) Side view of a solid particle emerging from a liquid with a receding contact line. 

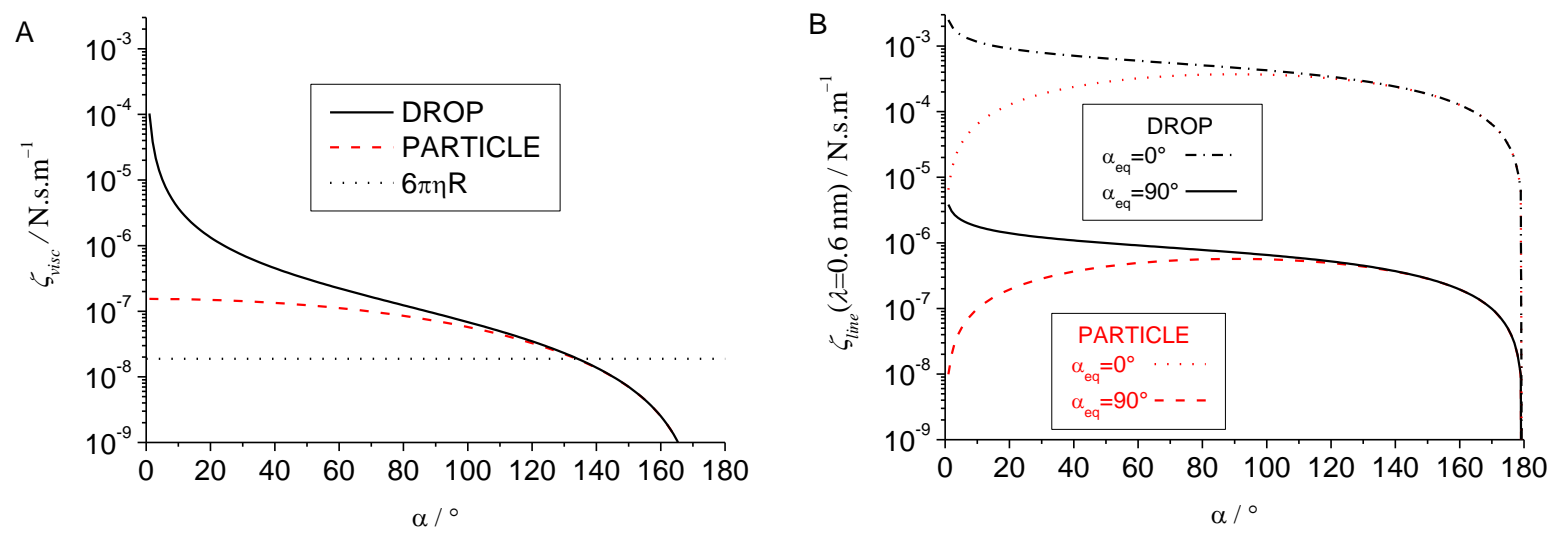

Figure $10 \mathrm{~A})$ Viscous friction coefficient as a function of the dynamic angle $\alpha$ for the drop and particle geometries $\left(\eta=10^{-3}\right.$ Pa.s, $\left.R=R_{0}\left(\alpha=180^{\circ}\right)=1 \mu \mathrm{m}\right)$. B) Line friction coefficients for drop and particle geometries for two different values of $\alpha_{e q}\left(\gamma=72 \mathrm{mN} / \mathrm{m}, R=R_{0}\left(\alpha=180^{\circ}\right)=1 \mu \mathrm{m}\right)$. 